Natalya V. Gubanova, Nina G. Orlova, Arthur I. Dergilev, Nina Y. Oparina and Yuriy L. Orlov*

\title{
Glioblastoma gene network reconstruction and ontology analysis by online bioinformatics tools
}

https://doi.org/10.1515/jib-2021-0031

Received August 31, 2021; accepted October 18, 2021; published online November 16, 2021

Abstract: Glioblastoma is the most aggressive type of brain tumors resistant to a number of antitumor drugs. The problem of therapy and drug treatment course is complicated by extremely high heterogeneity in the benign cell populations, the random arrangement of tumor cells, and polymorphism of their nuclei. The pathogenesis of gliomas needs to be studied using modern cellular technologies, genome- and transcriptomewide technologies of high-throughput sequencing, analysis of gene expression on microarrays, and methods of modern bioinformatics to find new therapy targets. Functional annotation of genes related to the disease could be retrieved based on genetic databases and cross-validated by integrating complementary experimental data. Gene network reconstruction for a set of genes (proteins) proved to be effective approach to study mechanisms underlying disease progression. We used online bioinformatics tools for annotation of gene list for glioma, reconstruction of gene network and comparative analysis of gene ontology categories. The available tools and the databases for glioblastoma gene analysis are discussed together with the recent progress in this field.

Keywords: drug search; gene networks; gene ontology; glioblastoma; medical genomics.

\section{Introduction}

Glioblastomas, along with astrocytomas, are the most common primary tumors of the central nervous system and make up approximately $60-70 \%$ of all pure brain tumors, challenging search for new therapy methods. Primary glioblastoma in $60 \%$ of cases occurs in people over 50 years old, while secondary glioblastoma is more characteristic for people under 45 years old [1, 2]. Glioblastoma is characterized by rapid infiltrative growth, the presence of foci of necrosis, a change in blood vessels. The lack of clear boundaries and the ability to relapse are a particular problem for surgical removal of the tumor, and for immunotherapy [3]. Primary

\footnotetext{
*Corresponding author: Yuriy L. Orlov, Novosibirsk State University, 630090 Novosibirsk, Russia; and The Digital Health Institute, I.M.Sechenov First Moscow State Medical University of the Russian Ministry of Health, 119991 Moscow, Russia, E-mail: y.orlov@sechenov.ru.https://orcid.org/0000-0003-0587-1609

Natalya V. Gubanova, Institute of Cytology and Genetics, Siberian Branch of the Russian Academy of Sciences, 630090 Novosibirsk, Russia, E-mail: nat@bionet.nsc.ru. https://orcid.org/0000-0003-2940-3287

Nina G. Orlova, Financial University under the Government of the Russian Federation, 119991 Moscow, Russia; and Moscow State Technical University of Civil Aviation, 125993 Moscow, Russia, E-mail: orlovanina2@mail.ru Arthur I. Dergilev, Novosibirsk State University, 630090 Novosibirsk, Russia, E-mail: arturd1993@yandex.ru Nina Y. Oparina, University of Gothenburg, 40530 Gothenburg, Sweden, E-mail: oparina@gmail.com
} 
central nervous system tumors are relatively rare and account for approximately $2 \%$ of all oncological diseases. However, tumors of the brain and spinal cord are the second most common form of malignancy in children after leukemia [4]. Glioblastoma has higher burden in survival of the patients with malignant brain tumors [5]. Despite the development in early detection, and treatment, 5-year glioblastoma survival only increased from 4 to $7 \%$ in past 4 decades [5]. Gliomas are the most common heterogeneous group of malignant tumors of the brain among the adult population, which differ in morphological characteristics, clinical course, and response to radio- and chemotherapy. The incidence of gliomas is 3-5: 100,000 of the adult population. In the treatment of glioblastoma with drugs, an obstacle is its resistance to a number of antitumor drugs initially, as well as the presence of a blood-brain barrier that negates the effectiveness of most chemotherapeutic agents and targeted drugs [3, 6].

For an object such as gliomas, it is necessary to conduct new studies based on modern cellular technologies, genome-wide technologies for high-throughput sequencing, and the integration of available information from international databases and genomic projects $[7,8]$.

The practical task was to construct (collect) a list of genes associated with the development of glioblastoma, analyze the categories of gene ontologies for such a list, and reconstruct the gene network. For key genes of the disease, obtained by analyzing the structure of the gene network, options for searching for drugs (substances that interact with this protein) will be considered.

There are open international databases on gene expression including glioblastoma (microarrays and sequencing data of GEO NCBI, http://www.ncbi.nlm.nih.gov/geoprofiles/), expression in various types of tumor cells (The Cancer Gene Atlas, cancergenome.nih.gov), gene expression for brain compartments (Allen Brain Atlas), protein interactions databases such as HPRD (http://hprd.org/), KEGG biochemical reactions (http://www.genome.jp/kegg/), Interactome (http://interactome.org/), sequenced tumor genomes, including gliomas and glioblastomas (https://cghub.ucsc.edu/). The Allen Institute has developed the Ivy Glioblastoma Atlas Project database (http://glioblastoma.alleninstitute.org/) according to patients with glioma. We demonstrate that these publicly available online bioinformatics tools can give helpful information for annotation of gene list for glioblastoma, reconstruction of gene network and comparative analysis of the related diseases. This approach for gene network analysis of list of gene names using online bioinformatics tools presents application of bioinformatics methods to annotation of complex human diseases.

\section{Methods}

Obtaining a list of genes associated with a hereditary predisposition to glioblastoma. The Internet resource OMIM (Online Mendelian Inheritance in Man) (https://omim.org/, access date 10.10.2021) was used to analyze the genes of Mendelian inheritance in humans [9]. Search for the keyword 'glioblastoma' gave 264 genes. As a result, 264 gene names (official gene symbols) were obtained (ADAM10, ADAM17, ADAM9, ADGRB1, etc., see the Supplement).

Next, we used DAVID (Database for Annotation, Visualization and Integrated Discovery) tool (https://david.ncifcrf.gov/ summary.jsp) [10] and the PANTHER (Protein ANalysis THrough Evolutionary Relationships) (http://pantherdb.org/) resource [11] for gene ontology analysis. Then we applied gene network reconstruction tools described in the next sections.

The workflow of the data processing is presented in Figure 1.

Figure 1 shows main steps of data processing. Data collection starts from the OMIM database (https://omim.org/) and GeneCards (https://www.genecards.org/) resources. At next step gene ontology analysis tools PANTHER and DAVID are used as well as g:Profiler (http://biit.cs.ut.ee/gprofiler/gost) online tool. Then STRING-DB (https://string-db.org) and GeneMANIA (https://genemania.org/) online applications help in the network reconstruction and visualization. Finally MalaCards (https://www.malacards.org/) and ToppGene (https://toppgene.cchmc.org/) allow find disease-disease interactions and prioritize genes in the list.

\section{Results}

We have analyzed gene ontology categories for the same gene list obtained by different online bioinformatics tools. DAVID [10] and the PANTHER resource [11] were used to estimate the categories of gene ontologies. 


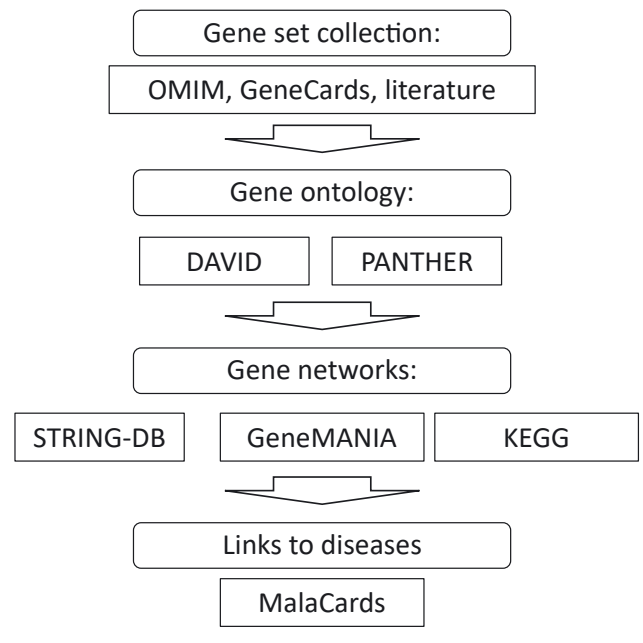

Figure 1: Workflow for information data processing for glioblastoma genes.

\subsection{Comparison of gene ontology categories}

A list of 264 human genes was loaded through the DAVID interface to search for relevant categories of gene ontologies for this group of genes. 255 identifiers were used next (the Functional Annotation Chart option).

The table was limited to values of normalized $p$-value $<0.00001$ (or, in power notation, $<1.0 \times 10^{-5}$ ) (Bonferroni correction). We deleted categories (table rows) for groups of less than eight genes, and Fold enrichment (observed number of genes/expected) less than 2.

As can be seen from Table 1, the most significant GO categories for glioblastoma genes are protein binding, positive regulation of protein phosphorylation, membrane location (according to cellular compartments - GOTERM_CC classification), and kinase activity. In general, according to the categories from the table,

Table 1: Categories of gene ontologies for glioblastoma genes according to DAVID

\begin{tabular}{llrrr}
\hline Category set & Term & \# genes & Fold & Bonferroni \\
\hline UP_KEYWORDS & Tumor suppressor & 24 & 12 & $1.3 \times 10^{-15}$ \\
UP_KEYWORDS & Phosphoprotein & 154 & 1.6 & $3.0 \times 10^{-13}$ \\
UP_KEYWORDS & Disease mutation & 71 & 2.5 & $1.2 \times 10^{-10}$ \\
GOTERM_MF_DIRECT & Protein binding & 164 & 1.5 & $4.2 \times 10^{-10}$ \\
GOTERM_BP_DIRECT & Negative regulation of cell proliferation & 26 & 5 & $1.8 \times 10^{-7}$ \\
INTERPRO & Protein kinase-like domain & 28 & 4.3 & $2.1 \times 10^{-7}$ \\
GOTERM_BP_DIRECT & Positive regulation of protein phosphorylation & 16 & 9.6 & $2.5 \times 10^{-7}$ \\
GOTERM_MF_DIRECT & Protein kinase binding & 24 & 5 & $2.6 \times 10^{-7}$ \\
UP_KEYWORDS & Kinase & 31 & 3.7 & $3.1 \times 10^{-7}$ \\
UP_KEYWORDS & Proto-oncogene & 18 & 6.7 & $4.4 \times 10^{-7}$ \\
UP_KEYWORDS & Cell cycle & 27 & 3.7 & $6.6 \times 10^{-6}$ \\
UP_KEYWORDS & Tyrosine-protein kinase & 12 & 9.5 & $1.5 \times 10^{-5}$ \\
INTERPRO & Protein kinase, catalytic domain & 24 & 4 & $1.8 \times 10^{-5}$ \\
UP_SEQ_FEATURE & Binding site:ATP & 25 & 3.9 & $3.2 \times 10^{-5}$ \\
GOTERM_MF_DIRECT & Kinase activity & 17 & 5.5 & $4.0 \times 10^{-5}$ \\
UP_SEQ_FEATURE & Active site:Proton acceptor & 27 & 3.5 & $6.8 \times 10^{-5}$ \\
GOTERM_BP_DIRECT & Protein phosphorylation & 24 & 4 & $7.7 \times 10^{-5}$ \\
GOTERM_MF_DIRECT & Protein kinase activity & 20 & 4.3 & $8.7 \times 10^{-5}$ \\
GOTERM_CC_DIRECT & Membrane & 56 & 2 & $9.6 \times 10^{-5}$ \\
\hline
\end{tabular}


membrane proteins and kinases play an important role, apparently associated with signal transduction into the cell. UP_KEYWORD (terms from UniProt (https://www.uniprot.org/) shows associations with tumors and kinase activity.

Gene ontologies were analyzed for the same list of genes using the PANTHER resource (Protein ANalysis THrough Evolutionary Relationships) [11]. Table 2 of ontologies for categories of biological processes was built using PANTHER.

In total, 238 identifiers were recognized from the same list of genes, the others were not recognized or could not be unambiguously mapped. In total, 20,595 genes were used in the PANTHER reference genome (15 October 2021). We limited the output to $p$-values up to $10^{-12}$ (after Bonferroni correction) and Fold enrichment greater than two to represent the most informative results. Table 2 shows that the most significant categories for glioblastoma genes are negative regulation of biological processes, developmental processes, generation of neurons, and cell proliferation.

The categories of processes related to the development of the nervous system are also presented (this is expected, since glioblastoma is a brain disease).

Further, the categories of gene ontologies for molecular functions and cellular compartments were calculated using PANTHER (Table 3). The threshold for the significance of the categories was taken already at the $10^{-3}$ level (after the Bonferroni correction).

The results for cellular components show that the most significant categories for glioblastoma genes are organelles, cell junction. It may be associated with signal transmission between cells and refers to neurons.

Thus, both DAVID and PANTHER for glioblastoma genes support the categories of gene ontologies of protein binding, membranes, cell adhesion, and intercellular contacts. Note that direct comparison of DAVID and PANTHER tools proved be limited due to different database version and genome annotation versions [12]. Other available resources for calculating gene ontologies - GeneOntology (http://geneontology.org/) and g:Profiler GOST (http://biit.cs.ut.ee/gprofiler/), which are not presented in this study, can also be used.

Table 2: Categories of gene ontologies for glioblastoma genes according to PANTHER (biological process).

\begin{tabular}{lrrr}
\hline GO categories (biological process complete) & \# genes & Fold enrich. & $\boldsymbol{p}$ value \\
\hline Negative regulation of biological process & 146 & 2.36 & $2.21 \times 10^{-25}$ \\
Negative regulation of cellular process & 137 & 2.40 & $2.20 \times 10^{-23}$ \\
Anatomical structure development & 136 & 2.28 & $5.27 \times 10^{-21}$ \\
System development & 123 & 2.47 & $7.48 \times 10^{-21}$ \\
Regulation of cell population proliferation & 75 & 3.81 & $3.21 \times 10^{-20}$ \\
Developmental process & 142 & 2.14 & $1.42 \times 10^{-19}$ \\
Regulation of signaling & 102 & 2.62 & $1.39 \times 10^{-17}$ \\
Regulation of cell differentiation & 67 & 3.75 & $3.90 \times 10^{-17}$ \\
Regulation of cell death & 69 & 3.63 & $4.97 \times 10^{-17}$ \\
Regulation of apoptotic process & 64 & 3.76 & $3.44 \times 10^{-16}$ \\
Cellular developmental process & 103 & 2.49 & $4.11 \times 10^{-16}$ \\
Regulation of programmed cell death & 64 & 3.68 & $9.59 \times 10^{-16}$ \\
Regulation of signal transduction & 91 & 2.66 & $3.02 \times 10^{-15}$ \\
Regulation of cellular component movement & 53 & 4.33 & $3.48 \times 10^{-15}$ \\
Nervous system development & 78 & 2.98 & $5.86 \times 10^{-15}$ \\
Regulation of epithelial cell proliferation & 32 & 7.93 & $1.10 \times 10^{-14}$ \\
Positive regulation of phosphorus metabolic process & 47 & 4.44 & $1.96 \times 10^{-13}$ \\
Immune system development & 40 & 5.04 & $1.21 \times 10^{-12}$ \\
Regulation of nervous system development & 33 & 6.31 & $1.69 \times 10^{-12}$ \\
Generation of neurons & 53 & 3.59 & $7.61 \times 10^{-12}$ \\
\hline
\end{tabular}


Table 3: Categories of gene ontologies for glioblastoma genes according to PANTHER (molecular functions and cellular compartments).

\begin{tabular}{lrrr}
\hline & \# genes & Fold enrich. & $p$ value \\
\hline GO categories - molecular function complete & & & \\
\hline Protein binding & 225 & 1.31 & $7.98 \times 10^{-13}$ \\
Enzyme binding & 68 & 2.76 & $2.84 \times 10^{-8}$ \\
Kinase binding & 36 & 3.94 & $1.54 \times 10^{-8}$ \\
Phosphotransferase activity alcohol group as acceptor & 31 & 3.76 & $1.57 \times 10^{-6}$ \\
Protein tyrosine kinase activity & 14 & 8.20 & $1.61 \times 10^{-5}$ \\
Transferase activity transferring phosphorus-containing groups & 34 & 3.09 & $2.78 \times 10^{-5}$ \\
Beta-catenin binding & 9 & 8.66 & $5.97 \times 10^{-3}$ \\
Growth factor receptor binding & 11 & 6.49 & $6.20 \times 10^{-3}$ \\
\hline GO categories - cellular component complete & & & \\
\hline Organelle lumen & 115 & 1.74 & $6.06 \times 10^{-8}$ \\
Cytosol & 111 & 1.72 & $3.16 \times 10^{-7}$ \\
Cell junction & 59 & 2.35 & $8.77 \times 10^{-7}$ \\
Cell projection & 63 & 2.23 & $1.75 \times 10^{-6}$ \\
\hline
\end{tabular}

\subsection{Reconstruction of gene networks for glioblastoma genes}

To reconstruct the gene network of interactions between glioblastoma genes, the resources of GeneMANIA (https://genemania.org/) and STRING-DB (https://string-db.org/) were used. The following Figure 2 shows the glioblastoma gene network reconstructed with GeneMANIA and STRING-DB.

In the centre of the network there are the genes (proteins) of glioblastoma, which have a large number of connections with other elements - PTRN, TP53, KRAS.

Consider the results of network reconstruction using STRING-DB (https://string-db.org/) for the same list of glioblastoma genes (Figure 2). It can be seen that the network is quite sparse, some objects (proteins) do not contact others. A central, strongly linked cluster of genes is highlighted.

In total, 243 genes from the list were recognized by STRING-DB. Statistics on STING-DB show that the network has a reasonably large number of connections (with a significance $<1.0 \times 10^{-16}$ ), the average degree of connectivity of the network node (protein) is 13.6, the clustering coefficient is 0.5. The drawing from the STING-DB was iteratively rebuilt with the removal of unrelated genes and the replacement of several colored connections between the nodes of the network - with one line (confidence), taking into account only experimentally proven interactions. Than we increase parameter 'confidence level' to keep only verified links in the network.

The figure shows several hubs of the network - the largest includes genes RBBP4, AKT1, MTOR, KRAS, STAT3, PTEN, several smaller, but also connected in a common network of clusters, the largest number of connections in the TP53 gene (a well-known oncogene) can be seen.

In general, the analysis of the structure of the gene network for glioblastoma genes shows the existence of a dense, connected, sufficiently large cluster of genes (network nodes) including known oncogenes, such as TP53.

Thus, standard online bioinformatics tools allow to model main parameters of gene network related to given disease. This approach is quite useful in distant education for medical students due to open access tools, fast visualization, interactive user interface not demanding programming. Integration of the results obtained by online bioinformatics tools and genetics data leads to novel knowledge in the study of complex oncological diseases. 


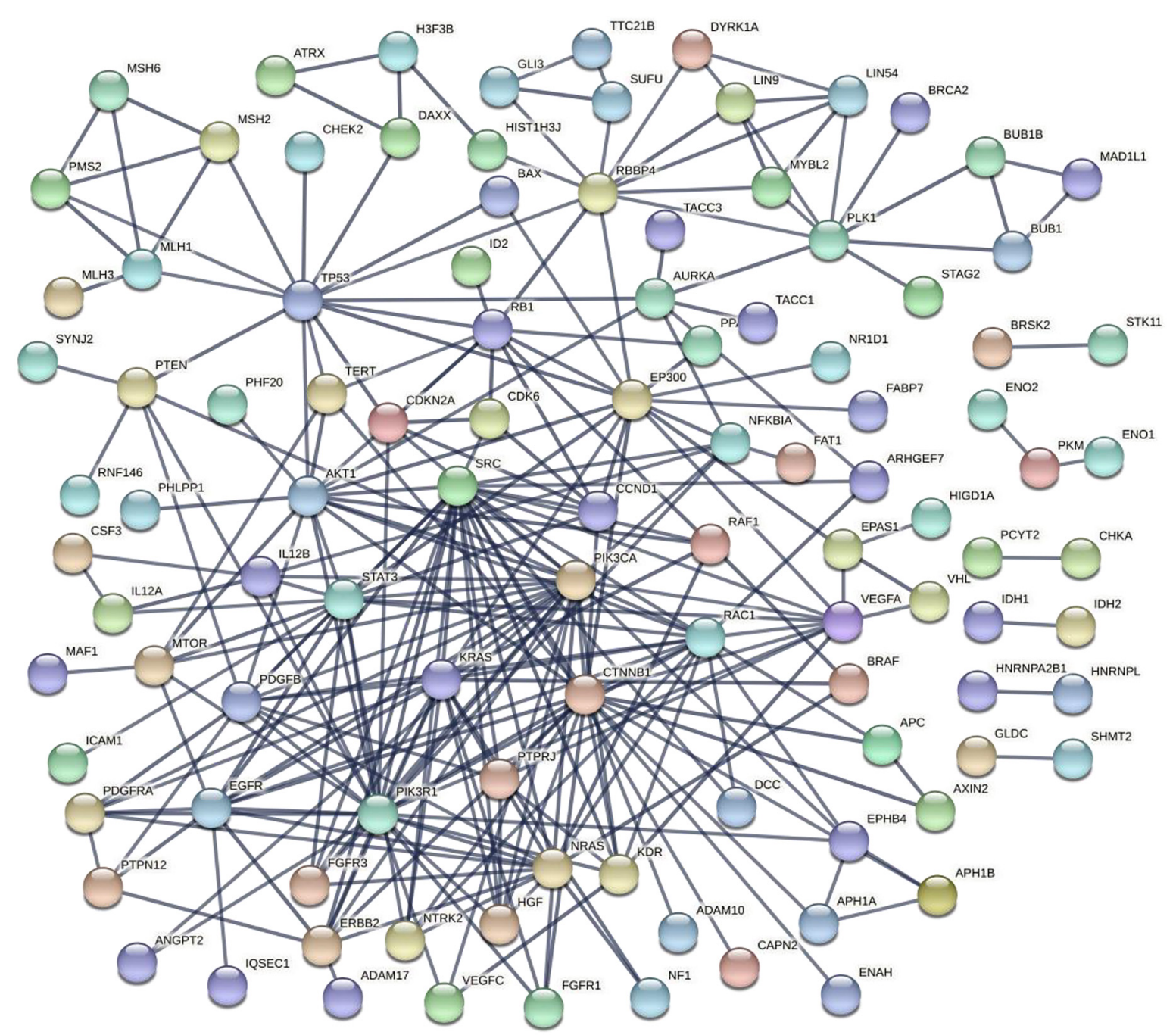

Figure 2: Gene network for glioblastoma genes reconstructed by STRING-DB.

\section{Related works}

Let's consider functional annotation of the glioblastoma genes using different data sources and their combinations.

According to GeneCards (genecards.org), the following 20 genes are the most significant.

Table 4 shows large fraction of non-protein coding genes in the list. The score in the table column show relevance of the gene to the search query to the literature database (https://www.genecards. org/Guide/Search\#relevance). Lager the GeneCards score, higher relevance to the disease is. GeneCards Inferred Functionality Scores (GIFtS) parameter shows gene functionality annotated in the databases (https://www.genecards.org/Guide/GeneCard\#GIFtS) [13]. Note that the hub genes in the network have high functionality, while RNA less annotated and included in standard gene network (in STRING-DB).

Prioritization of genes was performed using the resource ToppGene: Candidate gene prioritization (https://toppgene.cchmc.org) [14].

The genes presented were found to be associated with the following diseases (Table 5). 
Table 4: Gene list for glioblastoma by GeneCards.

\begin{tabular}{|c|c|c|c|c|c|}
\hline & Symbol & Description & Category & GIFtS & Relevance score \\
\hline 1 & TP53 & Tumor protein P53 & Protein coding & 52 & 52.29 \\
\hline 2 & EGFR & Epidermal growth factor receptor & Protein coding & 52 & 40.05 \\
\hline 3 & IDH1 & Isocitrate dehydrogenase (NADP(+)) 1 & Protein coding & 51 & 38.95 \\
\hline 4 & MSH2 & MutS homolog 2 & Protein coding & 48 & 33.76 \\
\hline 5 & MGMT & 0-6-Methylguanine-DNA methyltransferase & Protein coding & 48 & 31.36 \\
\hline 6 & ERBB2 & Erb-B2 receptor tyrosine kinase 2 & Protein coding & 52 & 29.57 \\
\hline 7 & MIR21 & MicroRNA 21 & RNA gene & 24 & 28.37 \\
\hline 8 & BRCA2 & BRCA2 DNA repair associated & Protein coding & 48 & 27.59 \\
\hline 9 & PTEN & Phosphatase and tensin homolog & Protein coding & 51 & 26.68 \\
\hline 10 & PIK3CA & $\begin{array}{l}\text { Phosphatidylinositol-4,5-bisphosphate 3-kinase } \\
\text { catalytic subunit alpha }\end{array}$ & Protein coding & 52 & 25.74 \\
\hline 11 & MIR221 & MicroRNA 221 & RNA gene & 21 & 24.00 \\
\hline 12 & MIR222 & MicroRNA 222 & RNA gene & 21 & 23.89 \\
\hline 13 & FGFR1 & Fibroblast growth factor receptor 1 & Protein coding & 53 & 22.81 \\
\hline 14 & MIR34A & MicroRNA 34a & RNA gene & 22 & 22.63 \\
\hline 15 & MIR296 & MicroRNA 296 & RNA gene & 17 & 22.51 \\
\hline 16 & H3-3A & $\mathrm{H} 3.3$ histone $\mathrm{A}$ & Protein coding & 34 & 21.76 \\
\hline 17 & MIR137 & MicroRNA 137 & RNA gene & 19 & 44.398 \\
\hline 18 & $\mathrm{IDH} 2$ & Isocitrate dehydrogenase (NADP(+)) 2 & Protein coding & 51 & 20.63 \\
\hline 19 & PPARG & Peroxisome proliferator activated receptor gamma & Protein coding & 51 & 20.37 \\
\hline 20 & MIR326 & MicroRNA 326 & RNA gene & 21 & 20.14 \\
\hline
\end{tabular}

Table 5: Diseases found by prioritization of shared genes to glioblastoma.

\begin{tabular}{llrr}
\hline No. & Description & Category & Score \\
\hline 1 & Glioma & $1.882 \times 10^{-25}$ & $9.333 \times 10^{-22}$ \\
2 & Pilocytic astrocytoma & $3.147 \times 10^{-25}$ & $1.561 \times 10^{-21}$ \\
$3-4$ & Adult pilocytic astrocytoma/childhood pilocytic astrocytoma & $1.829 \times 10^{-22}$ & $9.069 \times 10^{-19}$ \\
5 & Malignant glioma & $3.741 \times 10^{-21}$ & $1.855 \times 10^{-17}$ \\
6 & Mixed gliomas & $3.741 \times 10^{-21}$ & $1.855 \times 10^{-17}$ \\
7 & Neurofibromatosis 1 & $6.750 \times 10^{-21}$ & $3.348 \times 10^{-17}$ \\
8 & Malignant neoplasm of soft tissue & $2.560 \times 10^{-20}$ & $1.270 \times 10^{-16}$ \\
9 & Ganglioglioma & $4.231 \times 10^{-20}$ & $2.098 \times 10^{-16}$ \\
$10-11$ & Childhood oligodendroglioma/adult oligodendroglioma & $4.616 \times 10^{-20}$ & $2.290 \times 10^{-16}$ \\
12 & Sarcoma & $2.272 \times 10^{-19}$ & $1.127 \times 10^{-15}$ \\
\hline
\end{tabular}

One can see in the table relevant diseases categories related to brain, glioma types and soft tissues cancers.

The approaches of gene ontology analysis and gene network reconstruction using similar bioinformatics tools to search for glioma hub genes are published in series of papers. In recent work by [15] hub genes in the tumor microenvironment were identified using TCGA data. The hub genes for glioblastoma were identified by the Cytoscape tool (https://cytoscape.org/), and pathway enrichment analysis of the genes was performed using Database for Annotation, Visualization and Integrated Discovery (DAVID). Functional enrichment analysis identified set of upregulated and downregulated cross genes, which were mainly linked to immune response, inflammatory response, cell membrane, and receptor activity. Li and co-authors [16] used weighted gene co-expression network analysis to define hub genes.

Gene Set Enrichment Analysis (GSEA) identified further hub genes-related pathways. The Cancer Genome Atlas (TCGA) database was used to identify differentially expressed genes [17]. Then Gene Ontology and Kyoto 
Encyclopedia of Genes and Genomes (KEGG) analyses were used to determine the related functions and pathways of these genes.

Such analysis can lead to the identification of potential target genes and related drugs. Yang and Yang [18] recently analyzed glioma genes by GSEA, and the significantly enriched KEGG pathways involved in synapse signaling and oxytocin signaling pathways. The core molecules of GBM and the DrugBank database were assessed to identify 10 drugs included tetrachlorodecaoxide related to cancer and neuropsychiatric diseases [18].

\section{Conclusions}

After years of intensive research glioblastoma remains a dangerous disease with a low survival rate. The existing methods of therapy (chemotherapy, radio- and immunotherapy) can prolong the patient's life without remission and glioblastoma data integration and analysis can lead to promising therapeutic targets [19].

The study of the structure of the gene network shows a high connectivity of genes and their products. Glioma progression is strongly connected with different types of epigenetic phenomena, such as histone modifications, DNA methylation, chromatin remodeling, and aberrant microRNA [18]. Amid the epigenetic therapies, histone deacetylase inhibitors (HDACIs) and DNA methyltransferase inhibitors have been used for treating tumors [20].

Long non-coding RNAs, circular RNAs, and transcribed pseudogenes act as ceRNA (competing endogenous RNA) to regulate the expression of related genes by sponging the shared microRNAs [21]. Circular (circ)RNAs serve important roles in the development and progression of glioma and may have potential as therapeutic targets too [22].

A set of glioma-related genes obtained from the Comparative Toxicogenomics Database were predicted to be regulated by 15 miRNAs via the miRwalk 2.0 database [22]. Zhu and colleagues [23] provided a framework of workflow for potential therapeutic drug discovery and predicted 10 potential drugs for glioblastoma therapy. Effectiveness of drugs has to be tested on the experimental models [24]. Chemotherapy for glioblastoma remains ineffective due to insufficient penetration of therapeutic agents into the tumor [25]. Recent works show limits on the isotope distribution in tissues in a glioma xenograft animal model [26].

An analysis of the literature (PubMed) showed continued growth of publications on the topic - there are about 54 thousand publications up to date. Integration of large biological databases and re-analysis, combination of different tools allows keeping current structure of the disease description and annotation, as we have discussed at the international conferences series on computational genomics [27, 28]. Network analysis of interacting genes shows existing gene clusters in the network, and potential new targets for therapy [29]. Overall, such application of available bioinformatics tools for gene list analysis could serve as a standard for education [30, 31], allows to reveal novel features for cancer diseases. The network analysis complements analysis of microarray gene expression and transcriptomics data for glioblastoma research $[7,32]$. The network modelling using the described tools proved to be fruitful in other complex disease analysis, such as Parkinson disease, mental disorders, and diabetes [33, 34].

Acknowledgments: The authors are grateful to S. S. Kovalev, Institute of Cytology and Genetics SB RAS, and to V. A. Kononov, Agrarian and Technological Institute, RUDN University, Russia for science discussion and technical contribution.

Author contribution: NVG planned the study, NGO and AID did the computation and statistical analysis, NYO described disease network and drug target search, YLO planned the work and wrote the manuscript. All the authors have accepted responsibility for the entire content of this manuscript and approved its submission.

Research funding: The work was supported by the Ministry of Science and Higher Education of the Russian Federation via the Institute of Cytology and Genetics (state budget project No 0259-2021-0014).

Conflict of interest statement: Authors state no conflict of interest. 


\section{References}

1. Ohgaki H, Kleihues P. Genetic pathways to primary and secondary glioblastoma. Am J Pathol 2007;170:1445-53.

2. Bowman RL, Wang Q, Carro A, Verhaak RG, Squatrito M. GlioVis data portal for visualization and analysis of brain tumor expression datasets. Neuro Oncol 2017;19:139-41.

3. McGranahan T, Li G, Nagpal S. History and current state of immunotherapy in glioma and brain metastasis. Ther Adv Med Oncol 2017;9:347-68.

4. Franceschi S, Civita P, Pasqualetti F, Lessi F, Modena M, Barachini S, et al. Multiregional sequencing of IDH-WT glioblastoma reveals high genetic heterogeneity and a dynamic evolutionary history. Cancers 2021;13:2044.

5. Miller KD, Ostrom QT, Kruchko C, Patil N, Tihan T, Cioffi G, et al. Brain and other central nervous system tumor statistics. CA Cancer J Clin 2021;71:381-406.

6. Stavrovskaya AA, Shushanov SS, Rybalkina EY. Problems of glioblastoma multiforme drug resistance. Biochemistry 2016;81:91-100.

7. Babenko VN, Gubanova NV, Bragin AO, Chadaeva IV, Vasiliev GV, Medvedeva IV, et al. Computer analysis of glioma transcriptome profiling: alternative splicing events. J Integr Bioinform 2017;14:20170022.

8. Kovalev SS, Lieberfarb EY, Gubanova NV, Bragin AO, Galieva AG, Tsukanov AV, et al. Computer analysis of gene alternative splicing in glioma cell cultures by RNA-seq data. Vestnik NSU. Series: Information Technologies 2018;16:22-36.

9. Amberger JS, Bocchini CA, Scott AF, Hamosh A. OMIM.org: leveraging knowledge across phenotype-gene relationships. Nucleic Acids Res 2019;47:D1038-43.

10. Dennis G Jr, Sherman BT, Hosack DA, Yang J, Gao W, Lane HC, et al. DAVID: database for annotation, visualization, and integrated discovery. Genome Biol 2003;4:3.

11. Mi H, Muruganujan A, Casagrande JT, Thomas PD. Large-scale gene function analysis with the PANTHER classification system. Nat Protoc 2013;8:1551-66.

12. Talbott H, Hou X, Qiu F, Zhang P, Guda C, Yu F, et al. Transcriptomic and bioinformatics analysis of the early time-course of the response to prostaglandin F2 alpha in the bovine corpus luteum. Data Brief 2017;14:695-706.

13. Harel A, Inger A, Stelzer G, Strichman-Almashanu L, Dalah I, Safran M, et al. GIFtS: annotation landscape analysis with GeneCards. BMC Bioinf 2009;10:348.

14. Chen J, Bardes EE, Aronow BJ, Jegga AG. ToppGene suite for gene list enrichment analysis and candidate gene prioritization. Nucleic Acids Res 2009;37:W305-11.

15. Jiang Z, Shi Y, Zhao W, Zhang Y, Xie Y, Zhang B, et al. Development of an immune-related prognostic index associated with glioblastoma. Front Neurol 2021;12:610797.

16. Li C, Pu B, Gu L, Zhang M, Shen H, Yuan Y, et al. Identification of key modules and hub genes in glioblastoma multiforme based on co-expression network analysis. FEBS Open Bio 2021;11:833-50.

17. Xu B. Prediction and analysis of hub genes between glioblastoma and low-grade glioma using bioinformatics analysis. Medicine 2021;100:e23513.

18. Yang J, Yang Q. Identification of core genes and screening of potential targets in glioblastoma multiforme by integrated bioinformatic analysis. Front Oncol 2021;10:615976.

19. Corso CD, Bindra RS. Success and failures of combined modalities in glioblastoma multiforme: old problems and new directions. Semin Radiat Oncol 2016;26:281-98.

20. Uddin MS, Mamun AA, Alghamdi BS, Tewari D, Jeandet P, Sarwar MS, et al. Epigenetics of glioblastoma multiforme: from molecular mechanisms to therapeutic approaches. Semin Cancer Biol 2020;S1044-579X:30275-3.

21. Cen L, Liu R, Liu W, Li Q, Cui H. Competing endogenous RNA networks in glioma. Front Genet 2021;12:675498.

22. Wei B, Wang L, Zhao J. Circular RNA hsa_circ_0005114-miR-142-3p/miR-590-5p-adenomatous polyposis coli protein axis as a potential target for treatment of glioma. Oncol Lett 2021;21:58.

23. Zhu B, Mao X, Man Y. Potential drug prediction of glioblastoma based on drug perturbation-induced gene expression signatures. BioMed Res Int 2021;2021:6659701.

24. Raj D, Agrawal P, Gaitsch H, Wicks E, Tyler B. Pharmacological strategies for improving the prognosis of glioblastoma. Expert Opin Pharmacother 2021;22:2019-31.

25. Ahmed T, Liu FF, He C, Abbasi AZ, Cai P, Rauth AM, et al. Optimizing the design of blood-brain barrier-penetrating polymer-lipid-hybrid nanoparticles for delivering anticancer drugs to glioblastoma. Pharm Res 2021. https://doi.org/10 .1007/s11095-021-03122-9.

26. Gubanova NV, Tsygankova AR, Zavjalov EL, Romashchenko AV, Orlov YL. Biodistribution of 10B in glioma orthotopic xenograft mouse model after injection of L-para-boronophenylalanine and sodium borocaptate. Biomedicines 2021;9:722.

27. Orlov YL, Baranova AV, Hofestädt R, Kolchanov NA. Computational genomics at BGRSIISB-2016: introductory note. BMC Genom 2016;17:996.

28. Orlov YL, Baranova AV. Editorial: bioinformatics of genome regulation and systems biology. Front Genet 2020;11:625.

29. Orlov YL, Anashkina AA, Klimontov VV, Baranova AV. Medical genetics, genomics and bioinformatics aid in understanding molecular mechanisms of human diseases. Int J Mol Sci 2021;22:9962. 
30. Babenko VN, Bragin AO, Spitsina AM, Chadaeva IV, Galieva ER, Orlova GV, et al. Analysis of differential gene expression by RNA-seq data in brain areas of laboratory animals. J Integr Bioinform 2016;13:292.

31. Chen M, Harrison A, Shanahan H, Orlov Y. Biological big bytes: integrative analysis of large biological datasets. J Integr Bioinform 2017;14:20170052.

32. Gnanavel M, Murugesan A, Konda Mani S, Yli-Harja O, Kandhavelu M. Identifying the miRNA signature association with aging-related senescence in glioblastoma. Int J Mol Sci 2021;22:517.

33. Orlov YL, Galieva AG, Orlova NG, Ivanova EN, Mozyleva YA, Anashkina AA. Rekonstruktsiia gennoǐ seti bolezni Parkinsona dlia poiska genov-mishenei [reconstruction of gene network associated with Parkinson disease for gene targets search]. Biomed Khim 2021;67:222-30.

34. Saik OV, Klimontov VV. Bioinformatic reconstruction and analysis of gene networks related to glucose variability in diabetes and its complications. Int J Mol Sci 2020;21:8691.

Supplementary Material: The online version of this article offers supplementary material (https://doi.org/10.1515/jib-20210031). 